\title{
Erratum
}

\section{Ethical issues in publishing in predatory journals}

Lorraine E. Ferris ${ }^{* 1}$, Margaret A Winker ${ }^{2}$

'Dalla Lana School of Public Health, University of Toronto, Toronto, Canada

${ }^{2}$ Secretary, World Association of Medical Editors (WAME)

*Corresponding author: Lorraine.ferris@utoronto.ca

\section{Potential conflict of interest}

This is a correction for Biochemia Medica 2017;27(2):279-84. DOI: https://doi.org/10.11613/ BM.2017.029.

This Erratum corrects the section Potential conflict of interest, which was originally published. The publisher apologizes for this error.
Dr Ferris is the immediate past president of the World Association of Medical Editors (WAME) and Dr Winker is the current secretary (and a past president). WAME is a non-profit global voluntary association of editors of peer-reviewed medical journals. 